\title{
A CNN-Based Super-Resolution Technique for Active Fire Detection on Sentinel-2 Data
}

\author{
M. Gargiulo, D. A. G. Dell'Aglio, A. Iodice, D. Riccio, and G. Ruello \\ University of Naples "Federico II", Italy
}

\begin{abstract}
Remote Sensing applications can benefit from a relatively fine spatial resolution multispectral (MS) images and a high revisit frequency ensured by the twin satellites Sentinel2. Unfortunately, only four out of thirteen bands are provided at the highest resolution of 10 meters, and the others at 20 or 60 meters. For instance the Short-Wave Infrared (SWIR) bands, provided at 20 meters, are very useful to detect active fires. Aiming to a more detailed Active Fire Detection (AFD) maps, we propose a super-resolution data fusion method based on Convolutional Neural Network (CNN) to move towards the 10-m spatial resolution the SWIR bands. The proposed CNN-based solution achieves better results than alternative methods in terms of some accuracy metrics. Moreover we test the super-resolved bands from an application point of view by monitoring active fire through classic indices. Advantages and limits of our proposed approach are validated on specific geographical area (the mount Vesuvius, close to Naples) that was damaged by widespread fires during the summer of 2017.
\end{abstract}

\section{INTRODUCTION}

The remote sensing products are exploiting more and more in the earth monitoring because of the increasing number of satellites [1]. The European Space Agency has recently launched the twin satellites Sentinel-2 which can acquire global data for different applications such as risk management (floods, subsidence, landslide), land monitoring, water management, soil protection and so forth [2]. Sentinel-2 data are even useful in burnt area and active fire monitoring, using several algorithms [3]. A plethora of these is essentially based on the threshold of spectral indices involving Near-Infrared (NIR) and Short-Wave Infrared (SWIR) bands [4, 5, 6] that Sentinel-2 provides at spatial resolution of $10 \mathrm{~m}$ and $20 \mathrm{~m}$, respectively. Therefore, it is common to resort to the $20-\mathrm{m}$ resolution indices, by just downscaling the NIR band from $10 \mathrm{~m}$ to $20 \mathrm{~m}$. However, following this approach spatial information from NIR band would be lost. An alternative approach to enhance the AFD method using the Sentinel-2 images is to produce the Active Fire Indices (AFIs) by upscaling the SWIR bands from $20 \mathrm{~m}$ to $10 \mathrm{~m}$. Beyond the shadow of a doubt the main issue is the choice of the method to improve the spatial resolution of the SWIR bands. In general, Single Image Super Resolution (SISR) and Super Resolution Data Fusion (SRDF) methods are the two most popular ways to increase the spatial resolution of the images. The SISR methods do not use additional information from other sources, and they rely on spatial features of original image to increase its own resolution. On the other hand, SRDF methods (for instance, pan-sharpening) are based on the idea that the spatial information from other sources is useful to improve the spatial resolution of the original image [7]. In order to produce the 10-m AFIs from Sentinel-2 bands with SRDF methods, the use of all the highest spatial resolution bands is not very benefiting because of their smoke-sensitivity. The major contribution is derived from the NIR which is the only band we consider in the SRDF approaches.

The rest of the paper is organized as follows. Section 2 describes the study area and the picked dataset. Section 3 gives more details about the methodology, focusing on the proposed CNN-based super-resolution method, hereafter $S R N N_{+}$, on the spectral fire indices and on the considered accuracy metrics. Section 4 summarizes experimental results, placing more attention on the superresolved SWIR bands both in terms of visual inspection and numerical analysis while conclusions are drawn in Section 5.

\section{STUDY AREA AND DATASET}

The area under investigation is located at the Vesuvius (in Fig. 1), a volcano close to Naples,Italy. We are motivated by the choice of the study area since the presence of a natural park with a huge variety of flora and fauna considering its limited size [8]. At the beginning of July 2017, hundreds of wildfires ignited and damaged across the Italy country, whose the most serious were at Vesuvius. In 
fact, fires had been interesting the Vesuvius area for several days and the situation quickly became more dangerous due to adverse climatic conditions (winds and dry weather) [9]. The considered dataset is the Sentinel-2 Level-1C product acquired on 12th July 2017. As we can see in Fig. 1, the area under investigation is mainly covered by heavy smoke (Fig. 1-(b)) which reduces the usability of 10-m spectral information.

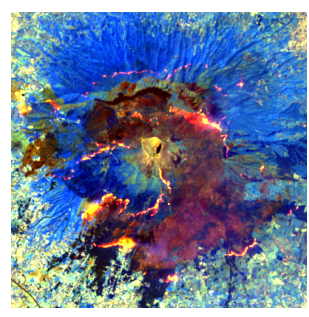

(a)

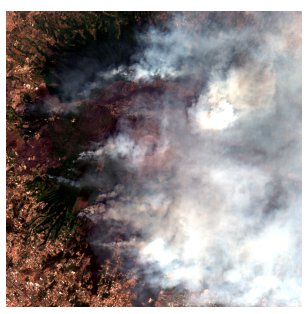

(b)

Figure 1: (a) false colour composite ( $\rho_{12}, \rho_{11}$ and $\rho_{8}$ bands) and (b) RGB image of Vesuvius .

\section{METHODOLOGY}

\subsection{Proposed CNN-based Super-Resolution Fusion}

Our goal is to improve the spatial resolution of SWIR bands using a Convolutional Neural Network $(\mathrm{CNN})$. CNNs have attracted an increasing interest in many remote sensing applications, like object detection [10], classification [11], pansharpening [12], and others, because of their capability to approximate complex non-linear functions, benefiting from the reduction in computation time obtained thanks to the GPU usage. On the downside the availability of a large amount of data is required for training. In this work we propose to use a relatively shallow architecture. This is composed by a cascade of $L=3$ convolutional layers. The first two are interleaved by Rectified Linear Unit (ReLU) activations that ensure fast convergence of the training process [11], and a linear activation function is considered in the last layer. The $l$-th $(1 \leq l \leq 3)$ convolutional layer, with $N$-band input $\mathbf{x}^{(l)}$, yields an $M$-band output $\mathbf{y}^{(l)}$

$$
\mathbf{y}^{(l)}=\mathbf{w}^{(l)} * \mathbf{x}^{(l)}+\mathbf{b}^{(l)} .
$$

In $l=1$ case, the $\mathbf{x}^{(l)}$ input is equal to the input, instead in $l=3$ case, the $\mathbf{y}^{(l)}$ is the output of the CNN. The tensor $\mathbf{w}$ is a set of $M$ convolutional $N \times(K \times K)$ filters, where a $K \times K$ is the receptive field, while $\mathbf{b}$ is a $M$-vector bias. These parameters, $\Phi_{l} \triangleq\left(\mathbf{w}^{(l)}, \mathbf{b}^{(l)}\right)$, are refined during the training phase. Further information about the CNN architecture can be found in [13]. In the supervised learning we need to generate a large amount of training samples, i.e. examples of inputs-target pairs. As reported in the pansharpening case [14] the training samples have to ensue the Wald's protocol, that means to consider as inputs the downsampled PAN-MS pairs and taking as corresponding output the original MS.

This approach has inspired our study where the highest spatial resolution bands play the role of the PAN and the MS is acted by SWIR. In our case we consider the training samples $\mathbf{x}^{(1)}=$ $(\tilde{\mathbf{x}}, \tilde{\mathbf{z}})$ as input to the network, where $\tilde{\mathbf{x}}$ and $\tilde{\mathbf{z}}$ are respectively the lower resolution version of the SWIR bands and of the 10-m bands provided by Sentinel-2, instead we consider the sharpened SWIR bands as output $\left(\mathbf{y}^{(3)}=\mathbf{x}\right)$. Furthermore, the cost function and the learning optimization algorithm are required in the learning phase. In more details we use the L1-norm as cost function, in place of the L2-norm, to be more effective in error back-propagation when the computed errors are very low [12]. Specifically, the loss is computed on the cost function by averaging over the training examples at each updating step of the learning process:

$$
L\left(\Phi^{(n)}\right)=\mathrm{E}\left[\left\|\mathbf{x}-\hat{\mathbf{x}}\left(\Phi^{(n)}\right)\right\|_{1}\right]
$$

where $\mathbf{x}$ represents the target and $\hat{\mathbf{x}}$ the output of the CNN, dependent on the learnable parameters $\left(\Phi^{(n)}\right)$. Instead, in this work we use the ADAM optimization method, an adaptive version of the Stochastic Gradient Descent (SGD), and it adapts the learning rates for each parameter of the CNN. This method requires very few tuning [15] and minimizes loss very speedily [16]. 


\subsection{Spectral Fire Indices}

The proposed model is evaluated, from the application point of view, by monitoring active fires through the computation of three different spectral indices (in Fig. 2), mainly used to this aim in literature $[17,18,19]$ because of their ease computing. The AFIs [17, 18] are defined on Sentinel-2 data as follows:

$$
A F I_{1}=\frac{\rho_{12}}{\rho_{8}} \quad A F I_{2}=\frac{\rho_{11}}{\rho_{8}} \quad A F I_{3}=\frac{\rho_{12}}{\rho_{11}}
$$

where $\rho_{8}$ is the $10-\mathrm{m}$ spatial resolution NIR band, centered at the wavelength of $0.834 \mu \mathrm{m}$; while $\rho_{11}$ and $\rho_{12}$ are the $20-\mathrm{m}$ SWIR bands, centered at $1.610 \mu \mathrm{m}$ and $2.190 \mu \mathrm{m}$, respectively. All of these bands represent the radiance data at top of the atmosphere. The choice of these indices is based on their physical properties. Specifically, the conditions $A F I_{1}>1$ and $A F I_{3}>1$ often occur in active fire; while the condition $A F I_{2}<1$ is verified near the fire fronts [19].
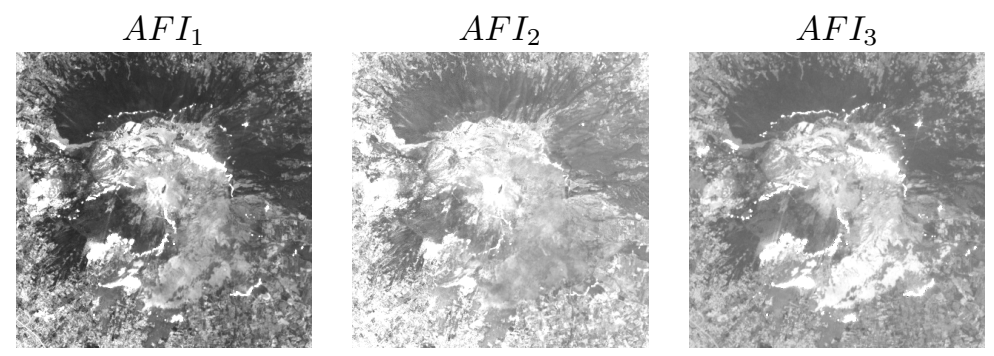

Figure 2: Active Fire Indices related to Vesuvius.

\subsection{Results Accuracy Metrics}

To evaluate the performance when the target image is available (in our case, at 20-m spatial resolution), the proposed method is compared to alternative methods using four reference metrics, commonly used for pansharpening [20]:

- Spectral Angular Mapper (SAM) the spectral distortion between pixel of reference image and estimated one [21];

- Universal Image Quality Index (UIQI, or Q-index), an image quality indicator introduced in $[22]$;

- Relative Dimensionless Global Error (as known as ERGAS) which reduces to the root mean square error (RMSE) in case of single band [7];

- High-frequency Correlation Coefficient (HCC), the correlation coefficient between the highpass components of two images [13].

For a full resolution analysis we consider the active fire monitoring application, and all the methods compete with each others in terms of binary classification. To this end we need to define a ground truth on which computing the main classification metrics. In this context, such ground truth is performed with a differential multi-temporal approach, based on a thresholding of the difference between two cloudily-free realizations of Normalized Difference Vegetation Index (NDVI) in two different date (before and after the fire event). This ground truth (GT) is affected by noise (or small bright pixels) and so we have used a morphological operator (opening) to erase this undesired noisy effect.

Thus, we compare this GT with the active fire maps obtained by thresholding the abovementioned spectral indices. In our case, the AFIs use the super-resolved bands with the different considered approaches. In particular we consider different thresholds on each of AFIs to match the best detection of the active fires. In order to evaluate the quality of the obtained binary maps, we have considered some metrics, typically used in classification task:

- Precision $(\mathrm{P})$ is the ratio between the correctly predicted positive observations and the total predicted positive ones;

- Recall $(\mathrm{R})$ is the ratio between the correctly predicted positive observations to the all observations in actual class;

- Intersection over Union (IoU) is the ratio between the overlapping area and the union area. The intersection and the union are computed on the predicted positive observations and the positives from the GT. 
It is worthwhile to remember that a high precision corresponds to a low false positive rate. In other words, higher the percentage of correctly predicted positive over the total predicted positive, higher precision. Instead, a high recall corresponds to a low false negative rate, that means higher recall higher detection rate.

\section{RESULTS AND DISCUSSION}

\subsection{Training Phase}

Given the lack of sufficient available input-output samples in the present context, we start from a pre-trained CNN solution [13] to train the network's parameters $\Phi^{n}$. In [13] a super-resolution technique is considered for $\rho_{11}$ band $\left(\mathbf{x}=\rho_{11}\right)$, and thus we extend to the $\rho_{12}$ band an equivalent solution $\left(\mathbf{x}=\rho_{12}\right)$. In particular, to create a pre-trained solution for this other band as well we use the identical dataset considered in [13]. After that, we fine-tune the weights of the CNN from Naples in two different dates, close to the target date (specifically June 27th and July 27th). This can be considered as a geographical fine-tuning, because we adapt the weights of the CNN on the geometric features of the study area. Then, we test this fine-tuned solution on the date under investigation (July 12th, 2017). Once left apart the target date for testing, $17 \times 17$ patches for training are uniformly extracted from two above-mentioned date in the remaining segments. Overall, 10k patches are collected from the considered dates and randomly partitioned in $80 \%$ for training phase and $20 \%$ for validation phase. The $8 \mathrm{k}$ training patches are grouped in 32 size mini-batches for the implementation of the ADAM-based training. The fine-tuned solution is considered better than the solution from scratch, when a large amount of data for training phase is not available, or when the computing power is not sufficient [23]. Eventually, we minimize the L1-norm cost function, defined in the Methodology section, on the training examples using the ADAM learning algorithm. Thus, we set the ADAM default values $\eta=0.002, \beta_{1}=0.9$, and $\beta_{2}=$ 0.999 , as reported in [24]. In this specific case, the training phase requires 200 epochs $(32 \times 200$ weight update) performed in few minutes using GPU cards, while the test can be done in real-time.

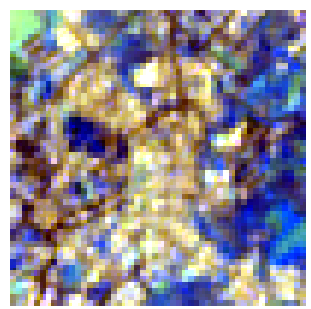

Nearest Neighbour

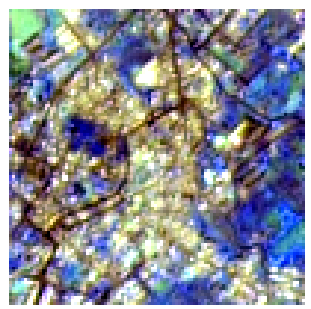

$\mathrm{HPF}$

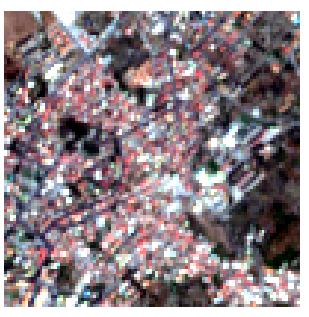

$\mathbf{Z}$

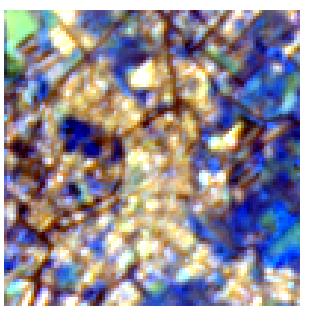

GS2-GLP

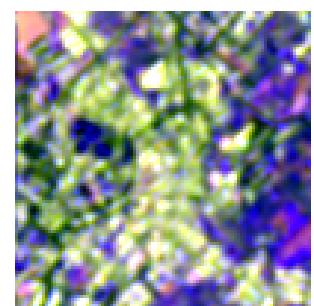

Bicubic

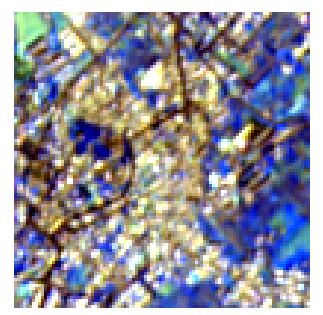

$S R N N_{+}$(Proposed)

Figure 3: Detail of the study area obtained by several super-resolution techniques and our proposal to underline the improvement in terms of spectral distortion. In the middle of the first row: $\mathbf{z}$ is only composed by RGB bands.

\subsection{Comparison between Super-Resolution Proposal and SISR/SRDF techniques}

In this section, $S R N N_{+}$is compared to a pre-trained CNN-based method (SRNN), three popular SRDFs adapted to the Sentinel-2/SWIR problem, including GS2-GLP [25], HPF [26] and PRACS [25], and even the SISRs, that are the Nearest Neighbour (NNI) and bicubic interpolation techniques. The numerical results obtained for the area of interest are reported in the left part of the Tab. 1. In the results we consider an average on the SWIR bands. In the top part of the table, the $S R N N_{+}$is compared to the SISR techniques, and the improvement is very remarkable in the HCC metric which deals with the fact that high frequency components are much affected by the super-resolution and are mostly localized on boundaries. Moving from the top to the bottom of the table, the proposed $S R N N_{+}$method compares favourably against classical fusion methods, which 
take information from the additional input band $\rho_{8}$. In the penultimate row it is given the performance of the pre-trained SRNN model and even in this case the $S R N N_{+}$performs slightly better results in terms of all the metrics at $20-\mathrm{m}$ resolution. As it can be seen the additional fine-tuning, although having few training patches, provide a further gain. To conclude this section we show in Figg. 4-3 some sample results (at 10-m without reference) which further confirm the effectiveness of the proposed method.

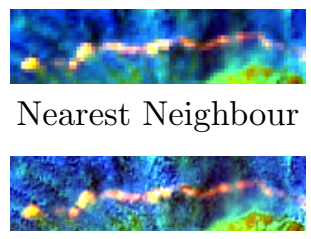

$\mathrm{HPF}$

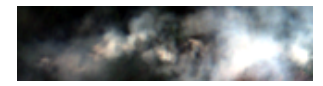

$\mathbf{Z}$

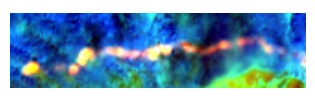

GS2-GLP

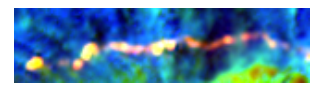

Bicubic

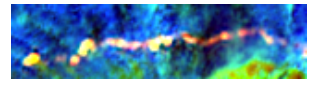

$S R N N_{+}$(Proposed)

Figure 4: Detail of the area under investigation obtained by several super-resolution techniques and our proposal to underline the improvement in terms of spectral distortion. In the middle of the first row: $\mathbf{z}$ is only composed by RGB bands that are affected by smoke presence (in the CNN input $\mathbf{z}$ is also composed by $\rho_{8}$ band.

\subsection{Comparison between Different AFIs and Maps}

Once active fire $(A F)$ is detected by considering the followed rules: $A F D=A F I_{k}>\alpha$, where $k \in\{1,2,3\}$, the performance is computed in terms of Precision, Recall and IoU and reported on the right-hand side of Tab. 1. The numerical results confirmed the effectiveness of the proposal, and the Fig.5-6 further confirm the superiority of the proposed method. As we can see in Tab. 1, the $S R N N_{+}$have the best performance in terms of precision metric. In particular, its values are much greater than those relating to the classic techniques, demonstrating that it benefits from the joint information obtained from the visible bands. The low false alarm rate aspect is well visible in Fig.6, where an urban detail included in the study area is shown. The Fig.6 only refers to $A F I_{2}$, but similar results are provided by the other indices analysed. On the other hand, both in terms of recall and IoU measures, the proposal have worse performance. We suppose this are mainly due to the ground truth used in validation, which probably over-estimates the areas interested by fires. In fact, as we can see in the central column of the figure 5, the $S R N N_{+}$AFIs better define these areas, resulting lighter and thinner then that ones obtained by other techniques. Furthermore we can observe from visual inspection that the boundaries are more evident considering $\rho_{12}$ and $\rho_{11}$ than $\rho_{12}$ and $\rho_{8}$. In general, even though this determines a low detection rate on the maps obtained by the $A F I_{3}$ with respect to $A F I_{1}$.

\begin{tabular}{l|cccc||ccc}
\hline Methods & SAM & Q-index & ERGAS & HCC & Precision & Recall & IoU \\
& $(0)$ & $(1)$ & $(0)$ & $(1)$ & $(1)$ & $(1)$ & $(1)$ \\
\hline NNI & 0.001960 & 0.9182 & 9.353 & 0.1355 & 0.8329 & 0.5773 & 0.5309 \\
Bicubic & 0.001964 & 0.9515 & 7.155 & 0.471 & 0.8387 & 0.5900 & 0.5471 \\
\hline HPF [26] & 0.064590 & 0.9405 & 8.150 & 0.2826 & 0.7799 & 0.5991 & 0.5476 \\
PRACS [25] & 0.001979 & 0.9535 & 7.057 & 0.5117 & 0.7993 & 0.5987 & 0.5497 \\
GS2-GLP [25] & 0.050190 & 0.9540 & 7.043 & 0.4694 & 0.8008 & $\mathbf{0 . 6 1 3 1}$ & $\mathbf{0 . 5 5 7 1}$ \\
\hline SRNN [13] & 0.001963 & 0.9688 & 5.943 & 0.6246 & 0.8373 & 0.5649 & 0.5158 \\
$S R N N_{+}$(Proposed) & $\mathbf{0 . 0 0 1 9 5 6}$ & $\mathbf{0 . 9 7 4 3}$ & $\mathbf{5 . 4 2 5}$ & $\mathbf{0 . 6 3 3 4}$ & $\mathbf{0 . 8 4 1 4}$ & 0.5642 & 0.5157 \\
\hline
\end{tabular}

Table 1: In the left part of the table: average results in terms of main metrics (at 20-m), typically used in pansharpening and super-resolution context. In the right part of the table: average results in terms of classification metrics.

\section{CONCLUSION}

In this work we propose the $S R N N_{+}$to further enhance the spatial resolution of the Sentinel-2 SWIR bands. For the specific goal (AFD) we fine-tune the weights of the CNN on the geographic study area and then we test the proposed approach both in terms of visual quality assessment and AFD capability. Eventually we show very promising results in terms of super-resolution metrics and even in AFD. The achieved results encourage us to exploit different architectural choices and/or learning strategies, and extending this approach to other remote sensing applications. 


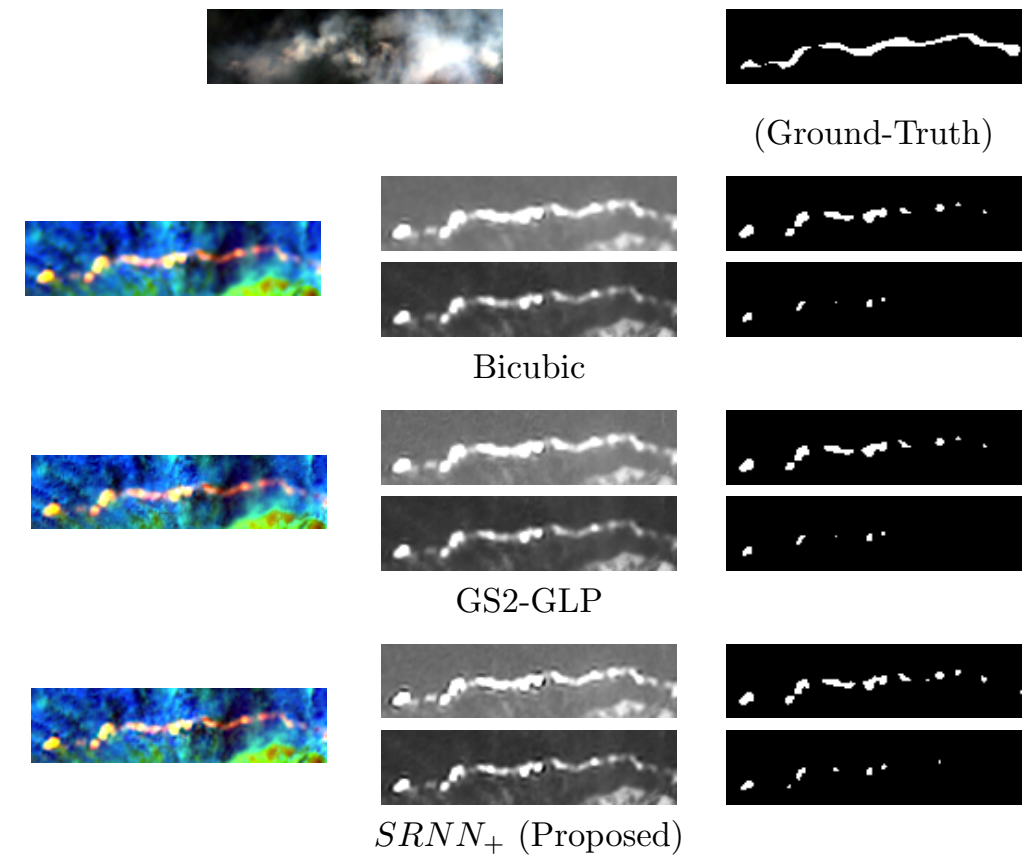

Figure 5: In the first row the RGB image in which we can observe the presence of the smoke and the ground truth. Then, from the second row to the bottom: in the first column false-RGB, in the second $A F I_{1}$ and $A F I_{3}$, in the third the respective Maps.

\section{REFERENCES}

1. Neha Joshi, Matthias Baumann, Andrea Ehammer, Rasmus Fensholt, Kenneth Grogan, Patrick Hostert, Martin Jepsen, Tobias Kuemmerle, Patrick Meyfroidt, Edward Mitchard, et al., "A review of the application of optical and radar remote sensing data fusion to land use mapping and monitoring," Remote Sensing, vol. 8, no. 1, pp. 70, 2016.

2. M. Drusch et al., "Sentinel-2: Esa's optical high-resolution mission for gmes operational services," Remote Sensing of Environment, vol. 120, no. Supplement C, pp. 25 - 36, 2012, The Sentinel Missions - New Opportunities for Science.

3. Astrid Verhegghen, Hugh Eva, Guido Ceccherini, Frederic Achard, Valery Gond, Sylvie Gourlet-Fleury, and Paolo Cerutti, "The potential of Sentinel satellites for burnt area mapping and monitoring in the Congo Basin forests," Remote Sensing, vol. 8, no. 12, pp. 986, 2016.

4. L Cicala, CV Angelino, N Fiscante, and SL Ullo, "Landsat-8 and Sentinel-2 for fire monitoring at a local scale: A case study on Vesuvius," in 2018 IEEE International Conference on Environmental Engineering (EE). IEEE, 2018, pp. 1-6.

5. José Pereira, Emilio Chuvieco, A Beudoin, and N Desbois, "Remote sensing of burned areas: A review. A review of remote sensing methods for the study of large wildland fires," Departamento de Geografa, Universidad de Alcal, pp. 127-184, 011997.

6. Yogesh Kant and K. V. S. Badarinath, "Studies on land surface temperature over heterogeneous areas using AVHRR data," International Journal of Remote Sensing, vol. 21, no. 8, pp. 1749-1756, 2000.

7. Lucien Wald, Data Fusion. Definitions and Architectures - Fusion of Images of Different Spatial Resolutions, Presses de l'Ecole, Ecole des Mines de Paris, Paris, France, 2002, ISBN 2-911762-38-X.

8. Lucio Mascolo, Maurizio Sarti, Ferdinando Nunziata, and Maurizio Migliaccio, "Vesuvius national park monitoring by COSMO-SkyMed PingPong data analysis," in ESA Special Publication, 2013, vol. 713.

9. G Bovio, M Marchetti, L Tonarelli, M Salis, G Vacchiano, R Lovreglio, M Elia, P Fiorucci, and D Ascoli, "Gli incendi boschivi stanno cambiando: cambiamo le strategie per governarli," Foresta - Rivista di Selvicoltura ed Ecologia Forestale, , no. 4, pp. 202-205, 2017.

10. Wei Guo, Wen Yang, Haijian Zhang, and Guang Hua, "Geospatial Object Detection in High Resolution Satellite Images Based on Multi-Scale Convolutional Neural Network," Remote Sensing, vol. 10, no. 1, pp. 131, 2018. 

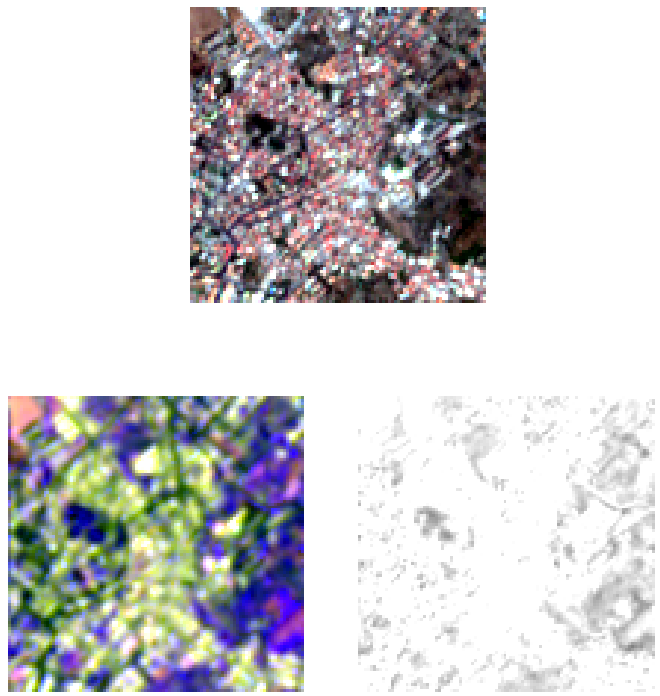

Bicubic
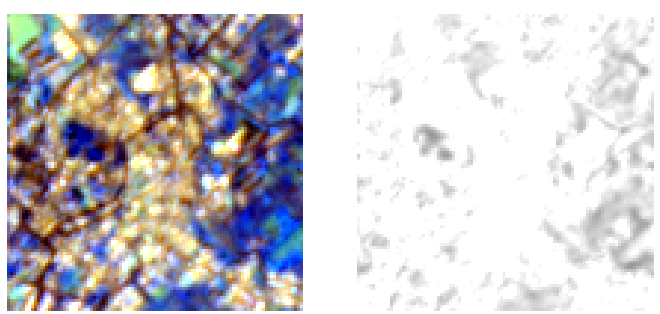

GS2-GLP

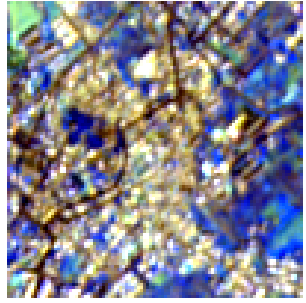

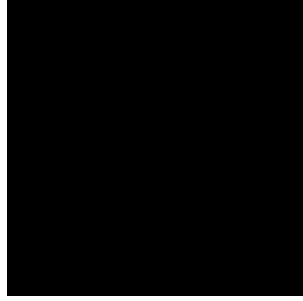

(Ground-Truth)
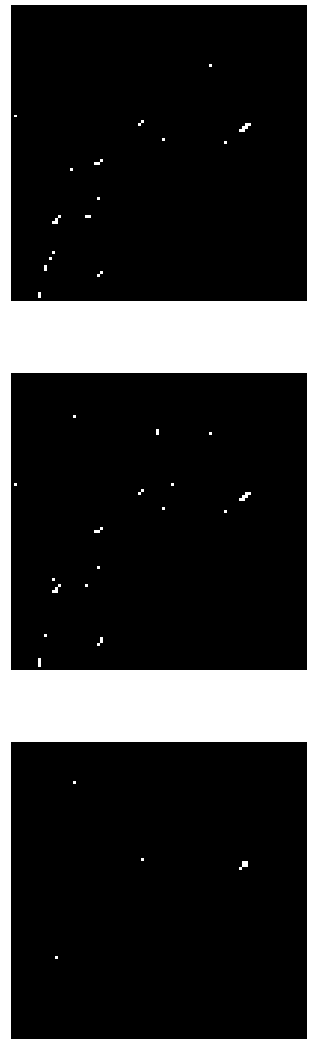

$S R N N_{+}$(Proposed )

Figure 6: In the first row the RGB image in which we can observe the absense of the smoke and the ground truth. Then, from the second row to the bottom: in the first column false-RGB, in the second $A F I_{2}$, and in the third the respective Map.

11. Alex Krizhevsky, Ilya Sutskever, and Geoffrey E. Hinton, "Imagenet classification with deep convolutional neural networks," pp. 1106-1114, 2012.

12. G. Scarpa, S. Vitale, and D. Cozzolino, "Target-adaptive cnn-based pansharpening," IEEE Transactions on Geoscience and Remote Sensing, vol. 56, no. 9, pp. 5443-5457, Sept 2018.

13. Massimiliano Gargiulo, Antonio Mazza, Raffaele Gaetano, Giuseppe Ruello, and Giuseppe Scarpa, "A CNN-Based Fusion Method for Super-Resolution of Sentinel-2 data," IGARSS, 2018.

14. Giuseppe Masi, Davide Cozzolino, Luisa Verdoliva, and Giuseppe Scarpa, "Pansharpening by convolutional neural networks," Remote Sensing, vol. 8, no. 7, pp. 594, 2016.

15. Kyle D Julian and Mykel J Kochenderfer, "Neural Network Guidance for UAVs," p. 1743, 2017.

16. Diederik P Kingma and Jimmy Ba, "Adam: A method for stochastic optimization," arXiv preprint arXiv:1412.6980, 2014.

17. Haiyan Huang, David Roy, Luigi Boschetti, Hankui Zhang, L Yan, Sanath Kumar, Jose GomezDans, and Jian Li, "Separability Analysis of Sentinel-2A Multi-Spectral Instrument (MSI) Data for Burned Area Discrimination," Remote Sensing, vol. 8, 112016. 
18. Wilfrid Schroeder, Patricia Oliva, Louis Giglio, Brad Quayle, Eckehard Lorenz, and Fabiano Morelli, "Active fire detection using Landsat-8/OLI data," Remote Sensing of Environment, vol. 185, 092015.

19. A Barducci, D Guzzi, P Marcoionni, and I Pippi, "Infrared detection of active fires and burnt areas: theory and observations," Infrared physics 8 technology, vol. 43, no. 3-5, pp. 119-125, 2002.

20. P Jagalingam and Arkal Vittal Hegde, "A review of quality metrics for fused image," Aquatic Procedia, vol. 4, pp. 133-142, 2015.

21. Luciano Alparone, Lucien Wald, Jocelyn Chanussot, Claire Thomas, Paolo Gamba, and Lori Mann Bruce, "Comparison of pansharpening algorithms: Outcome of the 2006 GRSS data-fusion contest," IEEE Transactions on Geoscience and Remote Sensing, vol. 45, no. 10, pp. 3012-3021, 2007.

22. Zhou Wang and A. C. Bovik, "A universal image quality index," IEEE Signal Processing Letters, vol. 9, no. 3, pp. 81-84, March 2002.

23. Nima Tajbakhsh, Jae Y Shin, Suryakanth R Gurudu, R Todd Hurst, Christopher B Kendall, Michael B Gotway, and Jianming Liang, "Convolutional neural networks for medical image analysis: Full training or fine tuning?," IEEE transactions on medical imaging, vol. 35, no. 5, pp. 1299-1312, 2016.

24. Sebastian Ruder, "An overview of gradient descent optimization algorithms," arXiv preprint arXiv:1609.04747, 2016.

25. Gemine Vivone, Luciano Alparone, Jocelyn Chanussot, Mauro Dalla Mura, Andrea Garzelli, Giorgio A Licciardi, Rocco Restaino, and Lucien Wald, "A critical comparison among pansharpening algorithms," IEEE Transactions on Geoscience and Remote Sensing, vol. 53, no. 5, pp. 2565-2586, 2015.

26. P.S. Chavez and J.A. Anderson, "Comparison of three different methods to merge multiresolution and multispectral data: Landsat TM and SPOT panchromatic," Photogramm. Eng. Remote Sens., vol. 57, no. 3, pp. 295-303, 1991. 